\title{
Nonresonant spin selection methods and polarization control in exciton-polariton condensates
}

\author{
M. Klaas, ${ }^{1, *}$ O. A. Egorov, ${ }^{1,2}$ T. C. H. Liew, ${ }^{3}$ A. Nalitov, ${ }^{4,5,6}$ V. Marković, ${ }^{1}$ H. Suchomel, ${ }^{1}$ T. H. Harder, ${ }^{1}$ S. Betzold, ${ }^{1}$ \\ E. A. Ostrovskaya, ${ }^{7}$ A. Kavokin, ${ }^{8,9,4}$ S. Klembt, ${ }^{1}$ S. Höfling, ${ }^{1,10}$ and C. Schneider ${ }^{1}$ \\ ${ }^{1}$ Technische Physik, Wilhelm-Conrad-Röntgen-Research Center for Complex Material Systems, Universität at Würzburg, Am Hubland, \\ D-97074 Würzburg, Germany \\ ${ }^{2}$ Institute of Condensed Matter Theory and Optics, Friedrich-Schiller-Universität Jena, Max-Wien-Platz 1, D-07743 Jena, Germany \\ ${ }^{3}$ Division of Physics and Applied Physics, School of Physical and Mathematical Sciences, Nanyang Technological University, \\ Singapore 637371, Singapore \\ ${ }^{4}$ School of Physics and Astronomy, University of Southampton, Southampton SO17 1BJ, United Kingdom \\ ${ }^{5}$ Science Institute, University of Iceland, Dunhagi 3, IS-107, Reykjavik, Iceland \\ ${ }^{6}$ ITMO University, St. Petersburg 197101, Russia \\ ${ }^{7}$ ARC Centre of Excellence in Future Low-Energy Electronics Technologies and Nonlinear Physics Centre, Research School of Physics and \\ Engineering, The Australian National University, Canberra, ACT 2601, Australia \\ ${ }^{8}$ Westlake University, 18 Shilongshan Road, Hangzhou 310024, Zhejiang Province, China \\ ${ }^{9}$ Institute of Natural Sciences, Westlake Institute for Advanced Study, 18 Shilongshan Road, Hangzhou 310024, Zhejiang Province, China \\ ${ }^{10}$ SUPA, School of Physics and Astronomy, University of St Andrews, St Andrews KY16 9SS, United Kingdom
}

(Received 17 September 2018; revised manuscript received 28 January 2019; published 4 March 2019)

\begin{abstract}
Bosonic condensates of exciton-polaritons are characterized by a well-defined pseudospin, which makes them attractive for quantum information schemes and spintronic applications, as well as the exploration of synthetic spin-orbit coupling. However, precise polarization control of coherent polariton condensates under nonresonant injection, the most important ingredient for such advanced studies, still remains a core challenge. Here, we address this problem and demonstrate unprecedented control of the pseudospin of an exciton-polariton condensate. The ultrafast stimulated scattering process allows the observation of completely spin-polarized condensates under highly nonresonant, circularly polarized excitation. This conservation of spin population translates, in the case of linearly polarized excitation, into an elliptically polarized emission. The degree of ellipticity can be controlled by varying the exciton-photon detuning and condensate density. Additionally, cavity engineering allows us to generate completely linearly polarized condensates with a deterministically chosen orientation. Our findings are of fundamental importance for the engineering and design of polaritonic devices that harness the spinor degree of freedom, such as chiral lasers, spin switches, and polaritonic topological insulator circuits.
\end{abstract}

DOI: 10.1103/PhysRevB.99.115303

\section{INTRODUCTION}

Spintronics is a research discipline where the intrinsic spin degree of freedom of an information carrier (such as an electron, exciton, or photon) is utilized to carry, store, and process information. In principle, it promises superior devices for integrated information architectures [1-3]. The ideal material platform for real-world applications is yet to emerge among traditional ferromagnetic competitors [4] and relatively new entrants such as carbon nanotubes [5], organic materials [6], semiconductor ferromagnets [7], and atomically thin layered materials [8]. They all seek to overcome the challenges imposed by temperature, intrinsic spin dephasing, and external spin control [9]. In particular the quantum extension of spintronics based on systems giving rise to quantum spin Hall physics is of interest, promising coherent, dissipationless transport of carriers in well-defined modes protected by topology [10]. Likewise, the spin of quantum states in well-controllable systems, such as single isolated defects or macroscopic condensates has been established as a

\footnotetext{
*martin.klaas@physik.uni-wuerzburg.de
}

prime candidate for a quantum bit, provided that initialization, coherent manipulation, and fast readout can be realized [11].

Condensates of exciton-polaritons (polaritons), bosonic quasiparticles consisting of excitons strongly coupled to photons in a semiconductor microcavity [12], have gained visibility as a novel hybrid photonic spinor platform. The solid-state environment allows for external engineering of devices via highly developed nanotechnology [13] and bosonic final-state stimulation promises ultralow thresholds [14,15] for highly coherent condensates $[16,17]$. State-of-the-art material engineering and technology advanced the field to enable condensation via electrical pumping $[18,19]$ and at room temperature [20-22].

Polaritons emerge from cavity photons which are strongly coupled to excitons and thus inherit the excitonic pseudospin as a degree of freedom [23]. The initialization of a polariton spin can be controlled by the polarization of an external resonant laser and probed through polarization of the cavity photoluminescence. While the dynamics of the Stokes vector on the Bloch sphere is determined by the intrinsic polarization anisotropy of the microcavity, it has been shown that very high degrees of polarization can be observed following resonant injection. Furthermore, by injecting on the lower 
polariton mode, stimulated bosonic scattering of polaritons has also been shown to conserve the polarization of the pump laser [15].

In the case of nonresonant injection, far above the exciton energy, polariton emission is typically unpolarized below the polariton condensation threshold due to loss of the pump laser polarization via fast spin relaxation in the long-lived exciton reservoir. In turn, a high degree of linear polarization is commonly observed as the system undergoes the transition into a condensate, which can be explained by the energy splitting due to random disorder in the studied samples or can build up along a specific crystal axis [24,25]. Polarization has also been shown to affect energy relaxation [26] and to switch in time-resolved studies [27]. Subject to circularly polarized, nonresonant pumping, approximately $40 \%$ conservation of circular polarization has also been observed [28,29], which is attributed to incomplete spin relaxation of the excited carriers, due to fast stimulated scattering [30]. A nearly fully polarized state has been achieved in Ref. [31]; however, the spin state is spontaneously chosen.

Indeed, the natural polarization sensitivity of most microcavity architectures utilized in the field yields an intrinsic dependence of the resonance energy on the polarization of the polariton state at a given wave vector, which has a striking similarity to the Rashba-type spin-orbit coupling in solid crystals. A direct manifestation of this effect is the observation of spin Hall phenomena in ballistically expanding polariton wave packets $[29,32,33]$. Similar to the solid-state counterpart [34], the possibility to engineer polarization effects, photonic crystal symmetries, and, most importantly, synthetic spinorbit coupling [35] enables the formation of polariton states with nontrivial Chern numbers. Comparable to carrier transport in quantum Hall edge modes, polariton propagation in such modes is predicted to be free of undesired backscattering [36-38] and was confirmed experimentally [39].

A possibility for utilizing the polariton pseudospin as an information carrier relies on our ability to control the polariton pseudospin in the microcavity with high accuracy. One method to preserve a certain degree of circular polarization of the driving pump has been introduced via a special ring-shaped pumping geometry in which the exciton reservoir has been separated from the condensate. In such pumping configurations, the formation of elliptically polarized condensates under linearly polarized pumping has also been observed [40,41].

In this work, we first demonstrate that circular polarization from a nonresonant excitation source can be fully conserved at the excitation spot, i.e., in the presence of the exciton reservoir in high-quality microcavities. We observe this effect in planar microcavities, as well as in circular micropillar structures. Secondly, we find that a linearly polarized pump will induce an elliptically polarized condensate, and we can tune the degree of ellipticity via the photon-exciton detuning and condensate density. Finally, we show that we can create fully linearly polarized condensates by etching nonrotationally symmetric pillars with an elliptic cross section. Hence, our work establishes nonresonant optical excitation in highquality samples as a tool for reliable preparation of a chosen spin state in a polariton condensate. (a)

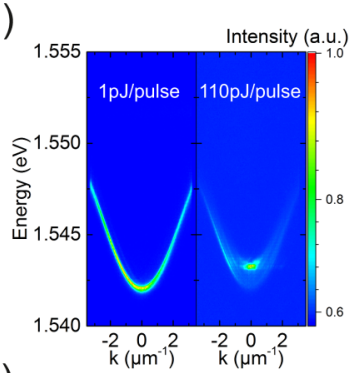

(b) (c)

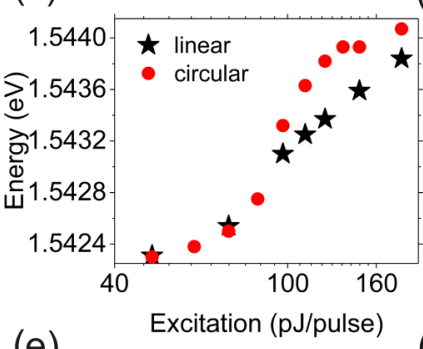

(e)

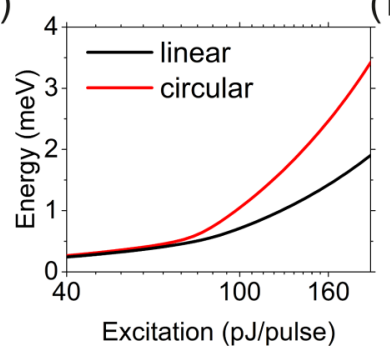

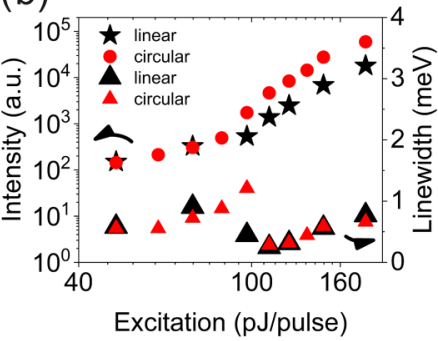

(d)

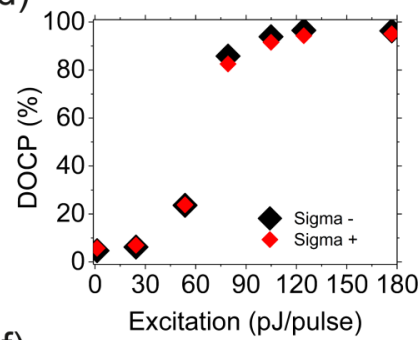

(f)

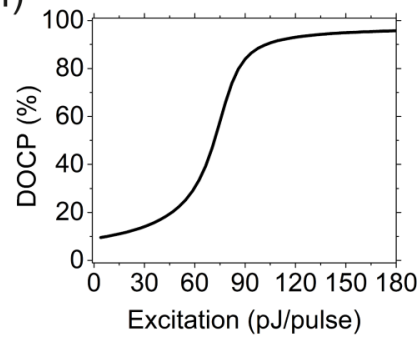

FIG. 1. (a) Dispersion below and above the condensation threshold at $1 \mathrm{pJ} /$ pulse and $110 \mathrm{pJ} /$ pulse input power. (b), (c) Input-output characteristics of the planar microcavity under nonresonant linearly (black) and circularly ( $\sigma^{+}$in red) polarized excitation. Intensity, linewidth, and energy were extracted from a Lorentzian fit of the ground state integrated around $k=0 \pm 0.1 \mu \mathrm{m}^{-1}$. (d) Circular polarization of the emission as a function of the input power (circularly polarized excitation). (e) Calculated energy shift as a function of the pump power, and (f) degree of circular polarization, calculated by utilizing the corresponding Boltzmann rate equation model (see text). The parameters for the modelling are $W=10^{-3} \mathrm{ps}^{-1}, \gamma_{c}=$ $(20)^{-1} \mathrm{ps}^{-1}, \gamma_{r}=5^{-1} \mathrm{ps}^{-1}, \gamma_{r s}=1 \mathrm{ps}^{-1}$.

\section{EXPERIMENT AND RESULTS}

The sample structure and experimental setup details can be found in the Supplemental Material [42]. Figure 1(a) depicts the momentum-resolved emission from the planar microcavity below and above the threshold for polariton condensation. The signal changes drastically from the thermally distributed population of the lower polariton branch to the massive occupation of a single energy and momentum state, characteristic of the exciton-polariton condensation. We fit the dispersions in Fig. 1 to the detuning of $-6.4 \mathrm{meV}$ (with a Rabi splitting of $10.1 \mathrm{meV}$ extracted from white-light reflectivity measurements). The details of the emission properties have been analyzed via a Lorentzian fit of the spectral line corresponding to the ground state. At a certain input power, a nonlinear increase of the emission intensity marks the condensation threshold [see Fig. 1(b)]. In the case of a 
circularly polarized excitation, we observe the presence of both condensate and low-density polaritons (due to the pulsed excitation) at approximately $100 \mathrm{pJ} /$ pulse, which explains the increased linewidth of the fit. For a linearly polarized excitation, no condensate is present at this pump power. For the same input powers, we observe a higher output intensity of the emission in the case of circularly polarized excitation. This result is compatible with the conclusion of the spindependent Boltzmann model considered below. Figure 1(b) reveals that transition to condensation is also reflected in the abrupt narrowing of the linewidth due to coherence buildup in the system [43]. Above the threshold, the linewidth increases due to interaction-induced loss of coherence [44]. Figure 1(c) presents a proof of preserved strong coupling conditions reflected in the characteristic blueshift of the emission above the threshold, originating from exciton-polariton and polaritonpolariton interactions in the system [45]. The difference for saturation of the blueshift of the two excitation polarizations is related to condensate density, which is higher in the case of circular polarization due to increased stimulated scattering and leads to a logarithmic blueshift shape. To understand the pronounced differences in emission characteristics under variously polarized excitation sources, we perform a detailed analysis of the spin population via the polarization properties of the photoluminescence. Figure 1(d) shows the degree of circular polarization of the emission for varying excitation powers across the polariton condensation transition (for the detuning of $-6.4 \mathrm{meV}$ between photon and exciton resonance). In this measurement, we excite the system with a circularly polarized laser and measure the degree of circular polarization [see the degree of circular polarization (DOCP) calculation example in the Supplemental Material [42]]. Below threshold, the emission is very weakly polarized, retaining approximately 5\% of DOCP. Above threshold, a DOCP of the condensate close to $100 \%$ can be observed. The DOCP therefore closely follows the excitation intensity with a clear threshold character, signifying the onset of the polarizationconserving stimulated scattering processes.

\section{MODELLING}

Both the increased blueshift observed under circularly polarized pumping and the dramatic increase in the degree of circular polarization can be understood in the framework of a semiclassical, spin-dependent Boltzmann model:

$$
\begin{aligned}
\frac{\mathrm{d} N_{r}^{ \pm}}{\mathrm{d} t} & =P^{ \pm}-W N_{r}^{ \pm}\left(N_{c}^{ \pm}+1\right)-\gamma_{r} N_{r}^{ \pm}-\gamma_{r s}\left(N_{r}^{ \pm}-N_{r}^{\mp}\right), \\
\frac{\mathrm{d} N_{c}^{ \pm}}{\mathrm{d} t} & =W N_{r}^{ \pm}\left(N_{c}^{ \pm}+1\right)-\gamma_{c} N_{c}^{ \pm},
\end{aligned}
$$

where $N_{c}{ }^{ \pm}$are the spin-polarized condensate populations and $N_{r}{ }^{ \pm}$are the reservoir of states with higher in-plane momentum. Here we distinguish between the particles with two different spin projections on the growth direction of the sample, namely " +1 " and " -1 "; $\gamma_{c}$ and $\gamma_{r}$ are the decay rates of the condensate and the reservoir, respectively, and $W$ is the rate at which reservoir particles scatter into the condensate. The spin-relaxation dynamics of this model is governed by the phenomenological constants $\gamma_{r s}$. This spin-flip scattering occurs in the reservoir owing to the TE-TM photonic mode splitting (or the longitudinal-transverse splitting of the exciton) [33]. Note that the splitting in the present sample is of the order of the linewidth. In the model Eq. (1), $P^{ \pm}$is the pumping rate created by an external incoherent optical beam. The two types of experiments with circularly and linearly polarized, nonresonant pumping are modelled by $P_{+}=P_{0}, P_{-}=0$, and $P_{+}=P_{-}=P_{0} / 2$, respectively.

The spin-dependent repulsive exciton-exciton interaction results in the blueshift of the condensate frequency $[24,45,46]$. For the experiment's relevant conditions the interaction between excitons with opposite spins can be neglected since the interaction between excitons of the same spin is substantially stronger [46]. Therefore the blueshift of the condensate in both configurations (i.e., circularly ' +1 ' and linearly polarized pumps) can be estimated as follows:

$$
\Delta \sim N_{r}^{+}+|X|^{2} N_{c}^{+},
$$

where $X$ is the exciton Hopfield coefficient. A linear scaling between the pumping power and the reservoir density was used as free parameter to obtain a quantitative agreement below and at the lasing threshold. Note that, at high pumping powers, observed dependence of the blueshift of a circularly pumped condensate saturates and the difference between the linear and circular pump cases diminishes. This can be qualitatively explained by the acceleration of spin relaxation through the Dyakonov-Perel mechanism due to disorder screening at high pumping powers. Indeed, in the limiting case of rapid spin relaxation, which is reached at high polariton densities, the spin-up and spin-down populations are equal regardless of the pump polarization. Figures 1(e) and 1(f) show the results obtained using this model, which qualitatively match the experimentally observed behavior under the assumption of sufficiently low transition rates between the spin states. The different blueshift of the emission for the same input power depending on the polarization of the pump can therefore be explained by the difference of interaction between equally and differently oriented spin populations. As has been shown previously [46], polaritons with parallel orientation of spins strongly repel each other, leading to an increased emission energy of the system.

This model possesses an analytical solution (for $\mathrm{N}_{c}^{ \pm} \gg 1$; see Supplemental Material [42] for a detailed derivation and explanation):

$$
S_{3}=\left\{\begin{array}{cc}
1 & \text { for } \quad P_{2} \geqslant P_{0}>P_{1} \\
\frac{S_{3 P} P_{0}}{\left(P_{0}-P_{t h}\right)} & \text { for } \quad P_{0}>P_{2},
\end{array}\right.
$$

where $S_{3}$ is the Stokes component for circular polarization. The threshold values are given by the following expressions:

$$
\begin{aligned}
& P_{1} \equiv P_{t h} \frac{\left(\gamma_{r}+2 \gamma_{r s}\right)}{\left[\left(1+S_{3 P}\right) \gamma_{r}+2 \gamma_{r s}\right]}, \\
& P_{2} \equiv \frac{P_{t h}}{\left(1-S_{3 P}\right)}, \quad P_{t h} \equiv \frac{2 \gamma_{r} \gamma_{c}}{W},
\end{aligned}
$$

where $P_{1}$ describes the condensation threshold of the spinor model (1) and $P_{2}$ is the critical pumping rate where the condensate becomes elliptically polarized. In accordance with this analytical solution, the condensation threshold (given by $\left.P_{1}\right)$ depends on the polarization rate $\left(S_{3 P}\right)$ of the optical pump beam. This value reaches a minimum for a circularly polarized 

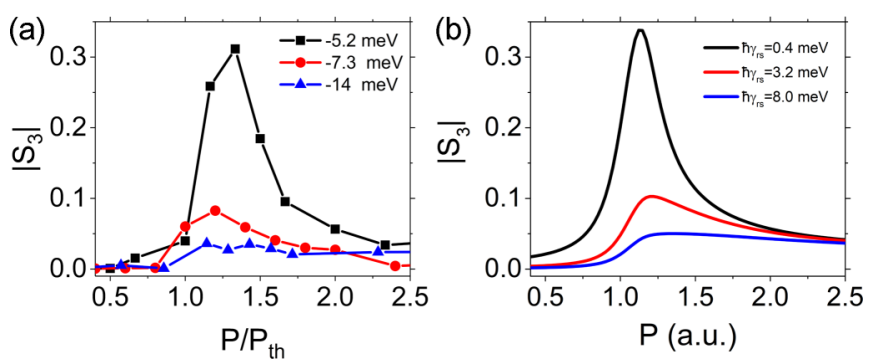

FIG. 2. (a) Degree of circular polarization characterized by the Stokes parameter $S_{3}$ of the condensate as a function of normalized input power for different photon-exciton detunings of the planar sample. (b) Modelled polarization $S_{3}$ of the condensate under the nearly linear polarization of the pump $\left(S_{3 P}=0.05\right)$ calculated for different values of the spin-scattering parameter $\gamma_{r s}$. The parameter $\gamma_{r s}$ depends on the photon-exciton detuning and can therefore explain the behavior of the condensate observed in panel (a).

pump $\left(S_{3 P}=1\right)$ whereas the condensate becomes circularly polarized since the second threshold diverges $\left(P_{2} \rightarrow \infty\right)$. We refer to the Supplemental Material [42] for further details.

Next, we study the polarization of our system subject to a linearly polarized excitation laser. The Stokes parameters $S_{0}, S_{1}, S_{2}$, and $S_{3}$ are extracted from a fit of the ground-state intensity evolution with retarder $\lambda / 4$ wave-plate orientation $(\Theta)[47]$.

Figure 2(a) shows values of the $S_{3}$ parameters as an indicator for the degree of circular polarization emerging in our system, plotted as a function of the excitation power and detuning between the photon and the exciton mode. As we increase the exciton content in our system, we observe higher degrees of circular polarization, in particular near the threshold. Further from the threshold, the circular degree of polarization increases with the excitation power to a certain point, from which it decreases rapidly.

To understand the observed occurrence of elliptical polarization, we need to account for a weak parasitic ellipticity of the pump, which is unavoidable in realistic experimental environments due to tight focusing of the laser beam. We introduce a slight imbalance between two circularly polarized components of the pump resulting in a weak elliptical polarization $S_{3 P}=0.05$ [40]. The steady-state solution (3) already provides the characteristic decay of the DOCP $\left(S_{3}\right)$ with growing pumping rate. However, it is necessary to go beyond the simplified steady-state approximation and consider experimentally relevant pulsed excitation with a pulse duration of about 2 ps.

To understand this behavior, we extend the Boltzmann model (see the Supplemental Material for more details [42]). In particular, we introduce two types of incoherent reservoirs [48]. The first "inactive" reservoir includes correlated electron-holes pairs and high-energy excitons, created by the external optical pump. The second subset, referred to below as the "active" reservoir, consists of excitons and exciton polaritons that fulfill energy- and momentum-conservation conditions to scatter directly into the condensate state and can only be replenished from the inactive reservoir injected by the pump. In full agreement with the experimental results, the polarization of the condensate shows a characteristic maximum above the condensation threshold and then it relaxes to some small value for growing pump rate [see Fig. 2(b)].

To illustrate the influence of the photon-exciton detuning, we calculated the Stokes parameter $S_{3}$ for different values of the spin-scattering rate $\gamma_{r s}$ in the "active" reservoir. We assume that the TE-TM photonic mode splitting [33] provides the main mechanism for the spin relaxation of the exciton-polaritons. This mechanism becomes feasible for exciton-polaritons in the high-momentum regions between the bottleneck [49] and inflection points of the lower-polariton dispersion branch. The photonic origin of this mechanism can explain the observed dependency of the condensate polarization on photon-exciton frequency detuning, since the photonic content of exciton-polaritons varies with the detuning. Furthermore, the typical momenta for the bottleneck polaritons (a)
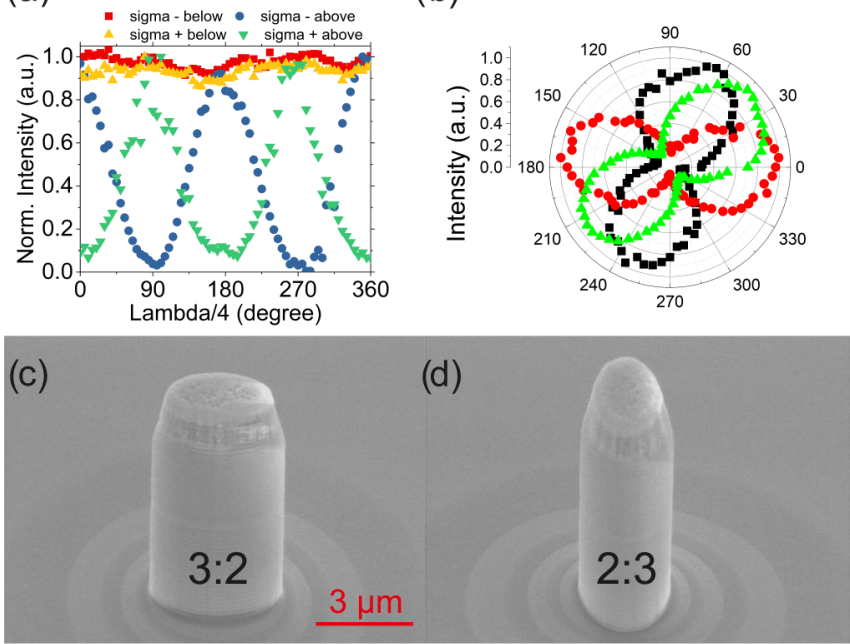

(e)

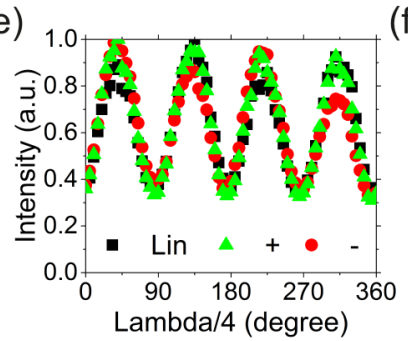

(b)

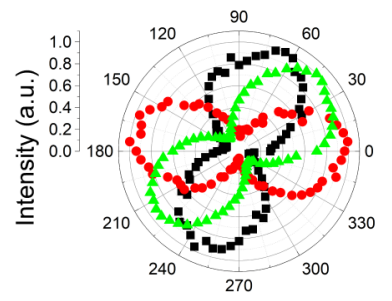

(f)

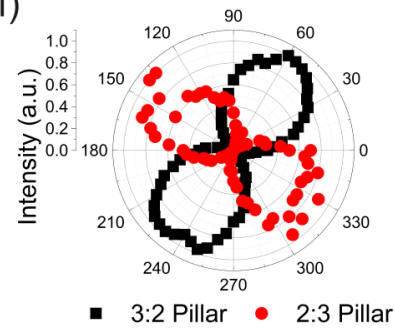

FIG. 3. (a) Stokes parameter resolved measurement of a $6 \mu \mathrm{m}$ micropillar microcavity below threshold under nonresonant $\sigma^{-}$excitation (red) and $\sigma^{+}$excitation (orange) and the same measurement technique above threshold ( $\sigma^{-}$blue, $\sigma^{+}$green), where a high degree of polarization conservation can be observed. (b) Linear polarization study of randomly chosen $2 \mu \mathrm{m}$ micropillars showing linearly polarized emission oriented along different axes, driven by a linearly polarized pump laser. (d) Scanning electron microscopy (SEM) images of an elliptical micropillar with (c) $3: 2$ aspect ratio and (d) $2: 3$ aspect ratio. (e) Stokes parameter resolved measurement for polarized excitations of an elliptical micropillar in the condensate regime: linear (black), $\sigma^{+}$(green) and $\sigma^{-}$(red). Similar Stokes parameters, indicating linear polarization, are observed for all pump polarizations in contrast to the system with rotational symmetry. The exciton-photon detuning is $-5 \mathrm{meV}$. (f) Linear polarization resolved intensity is measured with a $\lambda / 2$ wave plate for two elliptical systems with the long axis orthogonal to each other. 
are larger at negative exciton-photon detuning. In this case, the spin scattering becomes stronger, since the TE-TM photonic mode splitting scales as a square of the in-plane momentum $\left(\sim k^{2}\right)[23,33]$.

The arguments above suggest that the spin-scattering becomes larger for the pronounced negative photon-exciton detuning. As a consequence, the degree of circular polarization of the condensate becomes smaller, in full agreement with the experimental data. Figure 2 shows that the maximum of the $S_{3}$ curve vanishes for more negative photon-exciton detunings, while the condensate retains a moderate elliptical polarization for stronger pumping rates [cf. Figs. 2(a) and 2(b)].

\section{CONFINED SYSTEMS}

We have further extended our polarization study to etched micropillar cavities. Here, the same polarization conservation effect under circularly polarized pump takes place in circular micropillars with large diameters $(>3 \mu \mathrm{m})$. A typical measurement of a 6- $\mu \mathrm{m}$-diameter micropillar is shown below and above condensation threshold in Fig. 3(a). Surprisingly, in smaller micropillars, the circular polarization is not preserved to such a high degree. In turn, for a linearly polarized pump, we observe a linearly polarized emission from the condensate of varying, relatively high values (most micropillars in the range of 80\%), as shown in Fig. 3(b). The orientation of the linear polarization was found to vary arbitrarily throughout the investigated micropillars, but was always pinned along the same direction for repeated experiments carried out on the same pillar. This suggests, that the lithography process induced a slight asymmetrical shape on the pillars, which creates an intrinsic polarization splitting of two orthogonal, linearly polarized modes in the ground state. To validate this idea, we intentionally fabricated asymmetric micropillars of elliptical shape to induce a very high degree of linear polarization via the large energy splitting and to deterministically break the circular polarization conservation [50]. Figures 3(c) and 3(d) show the typical SEM pictures of elliptical micropillars with different orientations.

A full Stokes parameter measurement for a pillar with a long axis diameter of $2 \mu \mathrm{m}$ and an axis diameter ratio of $3: 2$ (i.e., an ellipticity $e=\sqrt{\frac{a}{b}}-1$ of 0.225 ) for the three different excitation polarizations is demonstrated in Fig. 3(d). The exact mode shapes for the elliptical micropillar are analytically calculated by using a time-independent Schrödinger equation approach for confined polariton modes detailed in the Supplemental Material [42]. The asymmetric spatial confinement induces a polarization splitting of the ground state of approximately $0.7 \mathrm{meV}$ (dependent on detuning), which favors the formation of linearly polarized polariton condensates. This manifests itself in linearly polarized emission regardless of the pump polarization, as evidenced by similar Stokes parameters or intensity evolution with retarder wave-plate position in Fig. 3(e), which is in striking difference to the prior results in symmetric systems [cf. Fig. 3(a)]. Moreover, Fig. 3(f) depicts a polar plot of the linear polarization of the condensates forming in two ellipses with the orthogonal orientation of their long axis. The linear polarization direction is, in this case, aligned along the long axis of the respective ellipse (in this case only the ground state is populated due to the high splitting), which reveals itself in the orthogonal alignment. Therefore, this technique allows, in principle, full linear polarization control of bosonic condensates by photonic engineering.

\section{CONCLUSION}

In conclusion, we have demonstrated the possibility to acquire extended control of the polarization of polariton condensates under nonresonant excitation. The fast relaxation dynamics of the spin, induced by bosonic stimulation, enables the observation of completely circularly polarized condensates which follow the circular polarization of the laser pump. A linearly polarized excitation at varying detunings and excitation strengths enables observation of emission with different degrees of ellipticity. Lastly, the fabrication of nonrotationally symmetric, elliptical micropillars introduces a linear polarization splitting in the system, which enables the observation of condensates that are linearly polarized along the direction of the long axis of the system. A high degree of polarization and the possibility to control the polarization orientation is a key ingredient for next-generation devices and experiments in polariton-based spintronics, quantum polaritonics, and the emerging field exploring topologically nontrivial phenomena via spin-orbit engineering in polaritonic devices [39].

\section{ACKNOWLEDGMENTS}

The authors would like to thank the State of Bavaria and the german research association (DFG) within the DFG project Schn1376 3-1 for financial support. E.A.O. acknowledges support by the Australian Research Council (ARC). A.N. acknowledges support from Icelandic Research Fund, Grant No. 196301-051 and from Russian Science Foundation, Grant No. 18-72-10110. T.H.H. gratefully acknowledges support by the Elite Network Bavaria within the doctoral training programme "Topological Insulators" (Tols 836315). Sample growth by S. Brodbeck, and technology support by M. Emmerling and A. Wolf is acknowledged. C. S. acknowledges discussions with T. Kiessling. T.C.H.L. was supported by the Singapore Ministry of Education Academic Research Fund Tier 2, Project No. MOE2017-T2-1-001.
[1] I. Žutić, J. Fabian, and S. Das Sarma, Rev. Mod. Phys. 76, 323 (2004).

[2] H. C. Koo, J. H. Kwon, J. Eom, J. Chang, S. H. Han, and M. Johnson, Science 325, 1515 (2009).

[3] S. Datta and B. Das, Appl. Phys. Lett. 56, 665 (1990).

[4] S. D. Bader and S. S. P. Parkin, Annu. Rev. Condens. Matter Phys. 1, 71 (2010).
[5] B. Zhao, I. Mönch, H. Vinzelberg, T. Mühl, and C. M. Schneider, Appl. Phys. Lett. 80, 3144 (2002).

[6] V. Dediu, M. Murgia, F. C. Matacotta, C. Taliani, and S. Barbanera, Solid State Commun. 122, 181 (2002).

[7] H. Ohno, Science 14, 951 (1998).

[8] D. Xiao, G.-B. Liu, W. Feng, X. Xu, and Wang Yao, Phys. Rev. Lett. 108, 196802 (2012). 
[9] D. D. Awschalom and M. E. Flatté, Nat. Phys. 3, 153 (2007).

[10] M. Z. Hasan and C. L. Kane, Rev. Mod. Phys. 82, 3045 (2010).

[11] T. D. Ladd, F. Jelezko, R. Laflamme, Y. Nakamura, C. Monroe, and J. L. O'Brien, Nature (London) 464, 45 (2010).

[12] C. Weisbuch, M. Nishioka, A. Ishikawa, and Y. Arakawa, Phys. Rev. Lett. 69, 3314 (1992).

[13] C. Schneider, K. Winkler, M. D. Fraser, M. Kamp, Y. Yamamoto, E. A. Ostrovskaya, and S. Höfling, Rep. Prog. Phys. 80, 016503 (2016).

[14] A. Imamoglu, R. J. Ram, S. Pau, and Y. Yamamoto, Phys. Rev. A 53, 4250 (1996).

[15] H. Deng, G. Weihs, D. Snoke, J. Bloch, and Y. Yamamoto, Proc. Natl. Acad. Sci. U. S. A. 100, 15318 (2003).

[16] S. Kim, B. Zhang, Z. Wang, J. Fischer, S. Brodbeck, M. Kamp, C. Schneider, S. Höfling, and H. Deng, Phys. Rev. X 6, 011026 (2016).

[17] M. Klaas, H. Flayac, M. Amthor, I. G. Savenko, S. Brodbeck, T. Ala-Nissila, S. Klembt, C. Schneider, and S. Höfling, Phys. Rev. Lett. 120, 017401 (2018).

[18] C. Schneider, A. Rahimi-Iman, N. Y. Kim, J. Fischer, I. G. Savenko, M. Amthor, M. Lermer, A. Wolf, L. Worschech, V. D. Kulakovskii, I. A. Shelykh, M. Kamp, S. Reitzenstein, A. Forchel, Y. Yamamoto, and S. Höfling, Nature (London) 497, 348 (2013).

[19] P. Bhattacharya, B. Xiao, A. Das, S. Bhowmick, and J. Heo, Phys. Rev. Lett. 110, 206403 (2013).

[20] J. D. Plumhof, T. Stoferle, L. Mai, U. Scherf, and R. F. Mahrt, Nat. Mater. 13, 247 (2014).

[21] K. S. Daskalakis, S. A. Maier, R. Murray, and S. Kena-Cohen, Nat. Mater. 13, 271 (2014).

[22] C. P. Dietrich, A. Steude, L. Tropf, M. Schubert, N. M. Kronenberg, K. Ostermann, S. Höfling, and M. C. Gather, Sci. Adv. 2, e1600666 (2016).

[23] A. Kavokin, J. J. Baumberg, G. Malpuech, and F. P. Laussy, Microcavities (Oxford University Press, Oxford, 2011).

[24] I. A. Shelykh, A. V. Kavokin, Y. G. Rubo, T. C. H. Liew, and G. Malpuech, Semicond. Sci. Technol. 25, 013001 (2010).

[25] V. G. Sala, F. Marsault, M. Wouters, E. Galopin, I. Sagnes, A. Lemaitre, J. Bloch, and A. Amo, Phys. Rev. B 93, 115313 (2016).

[26] G. Roumpos, C.-W. Lai, T. C. H. Liew, Yuri G. Rubo, A. V. Kavokin, and Y. Yamamoto, Phys. Rev. B 79, 195310 (2009).

[27] M. D. Martin, G. Aichmayr, L. Vina, and R. Andre, Phys. Rev. Lett. 89, 077402 (2002).

[28] H. Ohadi, E. Kammann, T. C. H. Liew, K. G. Lagoudakis, A. V. Kavokin, and P. G. Lagoudakis, Phys. Rev. Lett. 109, 016404 (2012).

[29] E. Kammann, T. C. H. Liew, H. Ohadi, P. Cilibrizzi, P. Tsotsis, Z. Hatzopoulos, P. G. Savvidis, A. V. Kavokin, and P. G. Lagoudakis, Phys. Rev. Lett. 109, 036404 (2012).

[30] I. Shelykh, K. V. Kavokin, A. V. Kavokin, G. Malpuech, P. Bigenwald, H. Deng, G. Weihs, and Y. Yamamoto, Phys. Rev. B 70, 035320 (2004).
[31] H. Ohadi, A. Dreismann, Y. G. Rubo, F. Pinsker, Y. del ValleInclan Redondo, S. I. Tsintzos, Z. Hatzopoulos, P. G. Savvidis, and J. J. Baumberg, Phys. Rev. X 5, 031002 (2015).

[32] A. Kavokin, G. Malpuech, and M. Glazov, Phys. Rev. Lett. 95, 136601 (2005).

[33] C. Leyder, M. Romanelli, J. Ph. Karr, E. Giacobino, T. C. H. Liew, M. M. Glazov, A. V. Kavokin, G. Malpuech, and A. Bramati, Nat. Phys. 3, 628 (2007).

[34] F. Reis, G. Li, L. Dudy, M. Bauernfeind, S. Glass, W. Hanke, R. Thomale, J. Schäfer, R. Claessen, Science 29, 10.1126 (2017).

[35] V. G. Sala, D. D. Solnyshkov, I. Carusotto, T. Jacqmin, A. Lemaître, H. Terças, A. Nalitov, M. Abbarchi, E. Galopin, I. Sagnes, J. Bloch, G. Malpuech, and A. Amo, Phys. Rev. X 5, 011034 (2015).

[36] A. V. Nalitov, G. Malpuech, H. Terças, and D. D. Solnyshkov, Phys. Rev. Lett. 114, 026803 (2015).

[37] T. Karzig, C.-E. Bardyn, N. H. Lindner, and G. Refael, Phys. Rev. X 5, 031001 (2015).

[38] C.-E. Bardyn, T. Karzig, G. Refael, and T. C. H. Liew, Phys. Rev. B 91, 161413(R) (2015).

[39] S. Klembt, T. H. Harder, O. A. Egorov, K. Winkler, R. Ge, M. A. Bandres, M. Emmerling, L. Worschech, T. C. H. Liew, M. Segev, C. Schneider, and S. Höfling, Nature (London) 562, 552 (2018).

[40] A. Askitopoulos, K. Kalinin, T. C. H. Liew, P. Cilibrizzi, Z. Hatzopoulos, P. G. Savvidis, N. G. Berloff, and P. G. Lagoudakis, Phys. Rev. B 93, 205307 (2016).

[41] L. Pickup, K. Kalinin, A. Askitopoulos, Z. Hatzopoulos, P. G. Savvidis, N. G. Berloff, and P. G. Lagoudakis, Phys. Rev. Lett. 120, 225301 (2018).

[42] See Supplemental Material at http://link.aps.org/supplemental/ 10.1103/PhysRevB.99.115303 for experimental and theoretical details.

[43] N. Wiener, Time Series (M. I. T. Press, Cambridge, Massachusetts, 1964).

[44] A. P. D. Love, D. N. Krizhanovskii, D. M. Whittaker, R. Bouchekioua, D. Sanvitto, S. Al Rizeiqi, R. Bradley, M. S. Skolnick, P. R. Eastham, R. André, and L. S. Dang, Phys. Rev. Lett. 101, 067404 (2008).

[45] C. Ciuti, V. Savona, C. Piermarocchi, A. Quattropani, and P. Schwendimann, Phys. Rev. B 58, 7926 (1998).

[46] M. Vladimirova, S. Cronenberger, D. Scalbert, K. V. Kavokin, A. Miard, A. Lemaitre, J. Bloch, D. Solnyshkov, G. Malpuech, and A. V. Kavokin, Phys. Rev. B 82, 075301 (2010).

[47] B. Schaefer, E. Collett, R. Smyth, D. Barrett, and B. Fraher, Am. J. Phys. 75, 163 (2007).

[48] K. G. Lagoudakis, F. Manni, B. Pietka, M. Wouters, T. C. Liew, V. Savona, A. V. Kavokin, R. Andre, and B. Deveaud- Pledran, Phys. Rev. Lett. 106, 115301 (2011).

[49] F. Tassone, C. Piermarocchi, V. Savona, A. Quattropani, and P. Schwendimann, Phys. Rev. B 56, 7554 (1997).

[50] S. Gerhardt, M. Deppisch, S. Betzold, T. H. Harder, T. C. H. Liew, A. Predojevic, S. Höfling, and C. Schneider, arXiv:1809.10459. 